\title{
Case Report: Tropheryma whipplei Hide in an AIDS Patient With Pneumocystis Pneumonia
}

OPEN ACCESS

Edited by:

Yanjiao Zhou,

UCONN Health, United States

Reviewed by:

Yaxsier De Armas,

Pedro Kouri National Institute of

Tropical Medicine (IPK), Cuba

Citra Fragrantia Theodorea,

University of Indonesia, Indonesia

*Correspondence:

Mengyan Wang

21618054@zju.edu.cn

Specialty section:

This article was submitted to Infectious Diseases - Surveillance,

Prevention and Treatment, a section of the journal

Frontiers in Public Health

Received: 02 February 2021

Accepted: 19 July 2021

Published: 13 August 2021

Citation:

Yan J, Zhang B, Zhang Z, Shi J, Liu S, Qi J and Wang M (2021) Case Report:

Tropheryma whipplei Hide in an AIDS

Patient With Pneumocystis

Pneumonia.

Front. Public Health 9:663093.

doi: 10.3389/fpubh.2021.663093

\section{Jun Yan, Binhai Zhang, Zhongdong Zhang, Jinchuan Shi, Shourong Liu, Jianjiang Qi and Mengyan Wang*}

Department II of Infectious Diseases, Xixi Hospital of Hangzhou, Zhejiang University, Hangzhou, China

Introduction: Pneumocystis pneumonia (PCP) is one of the most common opportunistic infections in HIV-infected patients. However, coinfection with Tropheryma whipplei is infrequent in AIDS patients with PCP.

Case Presentation: We report a 28-year-old male AIDS patient coinfected with $T$. whipplei and Pneumocystis jirovecii diagnosed in the bronchoalveolar lavage. After sulfamethoxazole-trimethoprim and meropenem treatment, the patient showed clinical improvement in 2 weeks.

Conclusion: Clinicians need to be alert to the occurrence of T. whipplei infection in AIDS patients with PCP and timely diagnosis and antibacterial treatments are essential. This case may help clinicians for timely diagnosis of the coinfection of $T$. whipplei and $P$. jirovecii in AIDS patients.

Keywords: Tropheryma whipplei, Pneumocystis pneumonia, HIV, case report, NGS

\section{INTRODUCTION}

Pneumocystis pneumonia (PCP) is one of the most common opportunistic infections in HIV-infected patients with high morbidity and mortality. The in-hospital mortality rate has been decreasing since the implementation of combination antiretroviral therapies and chemoprophylaxis. However, the reported mortality rate for PCP still ranged from 12.8 to $33.1 \%$ after antiretroviral therapies and chemoprophylaxis $(1,2)$. Tropheryma whipplei pneumonia is very infrequent and easy to be overlooked, especially in AIDS-PCP patients. T. whipplei can cause endocarditis, encephalitis, and other acute infections. T. whipplei was first detected in Whipple disease which was considered as a metabolic disease in the 20th century (3). In this study, we first report a case of T. whipplei coinfection with Pneumocystis jirovecii in an AIDS patient.

\section{CASE PRESENTATION}

A 28-year-old male patient had a dry cough, chest tightness after exercise, and shortness of breath for 1 week. He took oral cephalosporin for 3 days without any efficiency, and his symptoms got worse. On admission, leukoplakia in the mouth, little wet rales at the bottom of both lungs, and multiple swollen lymph nodes were found on clinical examination. His temperature was $37.6^{\circ} \mathrm{C}$. His blood test results showed that the white blood cell count was $6.52^{*} 10^{9 / L}$, with an elevation in neutrophil ratio of $72.7 \%$, high level of C-reactive protein (CRP) at $166 \mathrm{mg} / \mathrm{L}$ and serum amyloid protein A at $130 \mathrm{mg} / \mathrm{L}$, low level of oxygen partial pressure at $87.5 \mathrm{mmHg}$ and albumin 

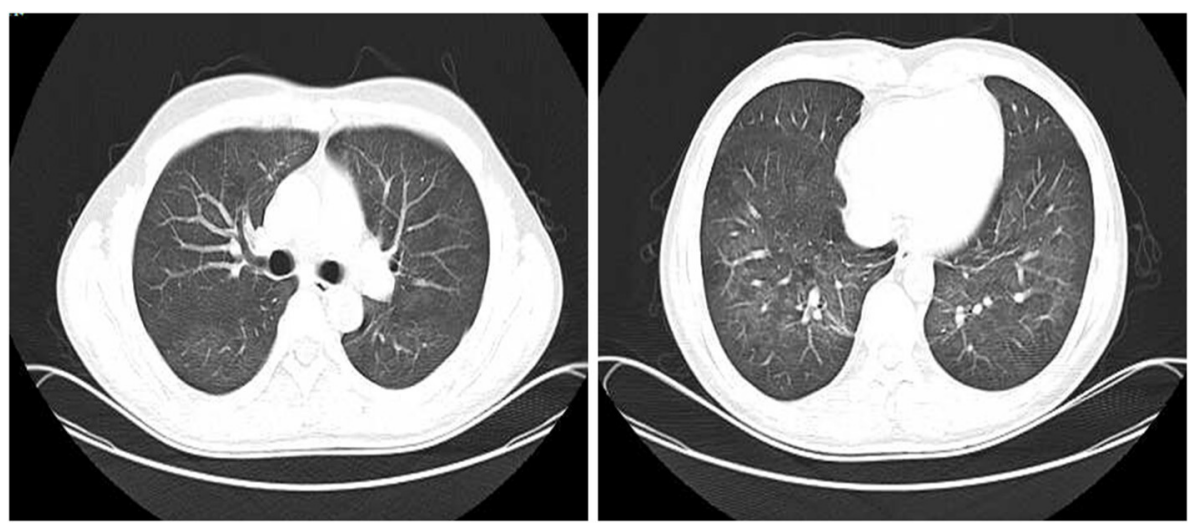

FIGURE 1 | CT of the lung revealed multifocal bilateral ground-glass opacities on admission.

at $30.3 \mathrm{~g} / \mathrm{L}$. Blood culture, antibody tests of Legionella pneumophila, Streptococcus pneumoniae, and Mycoplasma pneumoniae, and PCR-based detection of SARS-CoV-2 were negative. Cryptococcal capsular antigen, 1-3- $\beta$-D glucan detection, aspergillus galactomannan detection, and interferongamma release assays were negative. The viral load of HIV-RNA was $84,100 \mathrm{IU} / \mathrm{ml}$. CD4 ${ }^{+}$T-cell count was 11 cells $/ \mu \mathrm{l}$. CT of the lung revealed multifocal bilateral ground-glass opacities (Figure 1). According to the clinical diagnosis of PCP, the patient was treated with four pills of sulfamethoxazole-trimethoprim (sulfamethoxazole $1.2 \mathrm{~g}$, trimethoprim $240 \mathrm{mg}$ ) three times a day.

After treatment for 2 days (on day 3), the symptoms of the patient were more severe, and his temperature got to $39.9^{\circ} \mathrm{C}$. We performed a bronchoscopy on the patient, and it showed bronchial mucosa hyperemia and bronchial inflammatory changes. Nucleated cell counts of bronchoalveolar lavage (BAL) were $20 * 10^{6 / L}$. Cryptococcal antigen, $1-3-\beta$-D glucan detection, and culture of BAL were negative. Hexamine silver staining of $P$. jiroveci and PCR-based detection of cytomegalovirus and Mycobacterium tuberculosis were also negative. In addition, nextgeneration sequencing technologies (NGS) of BAL were carried out, the BAL was sent to a laboratory, and DNA was extracted for whole genomic sequencing, purified, and sonicated to a size of 100-150 bp. The DNA libraries were constructed followed by end repair, joint connection, no bias PCR amplification, and sequenced using the MGlseq-2000 platform after quality control. High-quality sequencing data were generated by removing lowquality reads. After removing human sequences, the remaining sequencing data were aligned to the bacterial, viral, fungal, and protozoan databases.

After treatment for 5 days (on day 6), CT of the lung revealed that it was worse than before (Figure 2). Results of NGS reported 205,658 unique reads of $P$. jirovecii, with coverage of identified genes $1.70 \%$ and 115 unique reads of $T$. whipplei, with coverage of identified genes $1.00 \%$ in BAL. The $P$. jirovecii-specific SYBR

Abbreviations: AIDS, Acquired immune deficiency syndrome; PCP, Pneumocystis pneumonia; BLAF, Bronchoalveolar lavage fluid; NGS, next-generation sequencing; CRP, C-reactive protein; PCR, polymerase chain reaction.
Green quantitative real-time PCR (qPCR) assay targeting a 301 bp fragment using a CFX96 Real-Time System was performed. The forward primer ( $\mathrm{pH} 207$ 5-ACAAATCGGACTAGGATAT AGCTGGT-3) and the reverse primer pAZ102-E were used to detect the mtLSUrRNA gene (4), and the copy number of $P$. jiroveci was 4,787.36 copies/ml. For specific T. whipplei qPCR, the specimen was tested by using Twhi3F (5-TTGTGTATT TGGTATTAGATGAAACAG-3), Twhi3R (5-CCCTACAATATG AAACAGCCTTTG-3) primer pair, and the specific TaqMan probe Twhi3 (6-FAM-GGGATAGAGCAGGAGGTGTCTGTC TGG-TAMRA). When the specimen was positive in this assay, the result was confirmed by a second qPCR by using Twhi2F (5-TGAGGATGTATCTGTGTATGGGACA-3) andTwhi2R (5TCCTGTTACAAGCAGTACAAAACAAA-3) primer set, and the Twhi2 probe (6-FAM-GAGAGATGGGGTGCAGGACAG GG-TAMRA) (5). The copy number of T. whipplei was $11,717.3$ copies/ml. Considering the progressed condition of the patient, meropenem $1 \mathrm{~g} \mathrm{q} 8 \mathrm{~h}$ and caspofungin $70 \mathrm{mg}$ per day were added for treating the infections of T. whipplei and $P$. jirovecii. Methylprednisolone $40 \mathrm{mg}$ BID was added due to the severe symptoms. On day 12, there was a clinical improvement, and CT of the lungs showed obviously better than before (Figure 3), and the patient was discharged on day 16 .

\section{DISCUSSION AND CONCLUSIONS}

A previous study had found that T. whipplei could easily colonize in the lungs of HIV individuals by analyzing bacterial gene sequences in BAL (6). An AIDS patient with T. whipplei pneumonia had been reported before, and Stein et al. suggested that T. whipplei should be considered in the differential diagnosis of pneumonia in AIDS patients (7). In this study, we reported the coinfection of T. whipplei and $P$. jirovecii pneumonia, which frequently had similar symptoms of fever, cough, and dyspnea (8-10), and a previous case-control study revealed that $P$. jirovecii was frequently associated with the T. whipplei pneumonia (11).

As we all know, lung infections by opportunistic or virulent pathogens are the major reasons that cause high mortality in AIDS patients. However, it is difficult to diagnose 

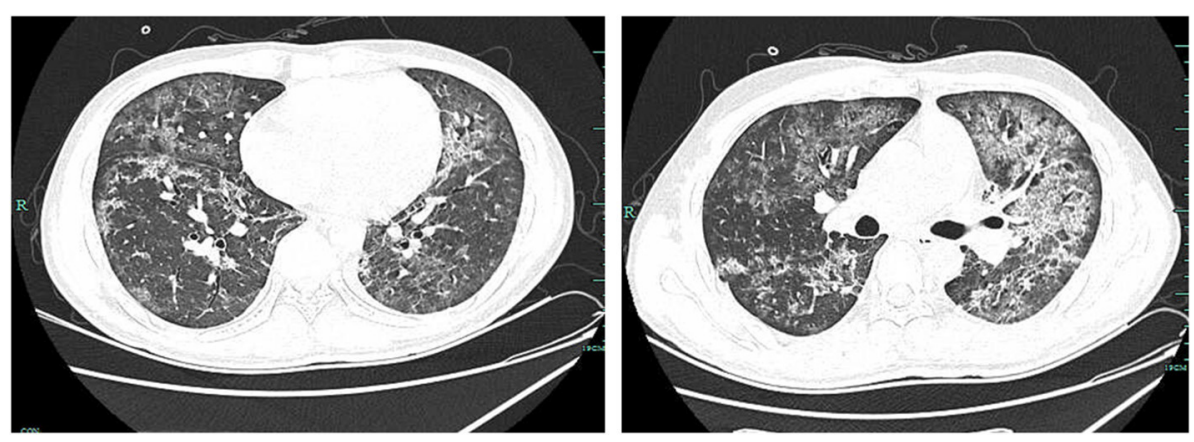

FIGURE 2 | CT of the lung revealed that multiple infections occurred in bilateral lungs, and they were scattered with diffuse patchy, ground glass-like, and high density of shadows on day 6 .

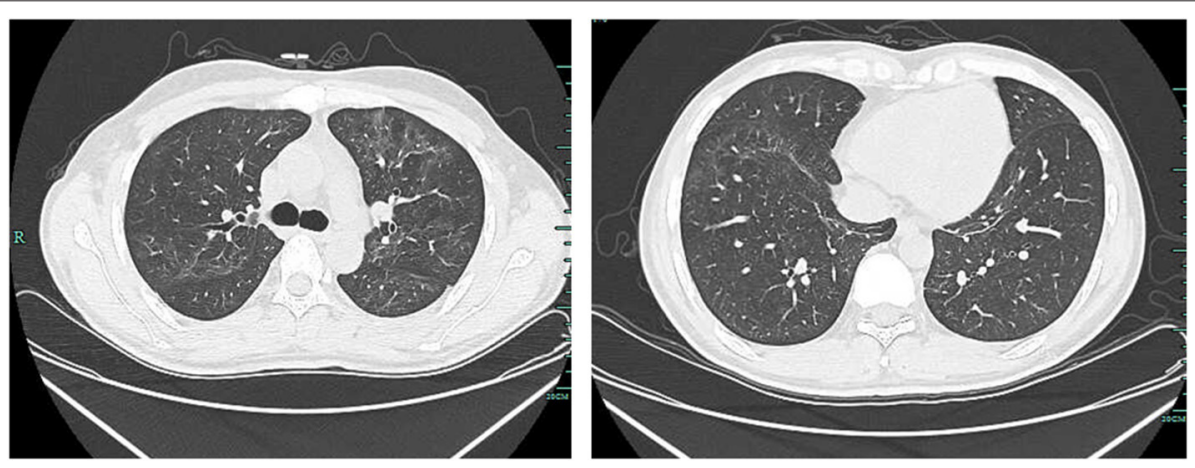

FIGURE 3 | CT of the lung showed that multiple infections occurred in bilateral lungs, and they were better, slightly diffuse, and with a ground glass-like high density of shadows on day 12 .

specific pathogens of lung infection. Due to the difficulty of culture, the diagnosis methods of $P$. jirovecii included direct cytochemical staining, immunofluorescent staining with monoclonal antibodies, and molecular methods such as PCR, with no ideal specificity and sensitivity (12). Similar to $P$. jirovecii, the culture of T. whipplei is difficult, which may not be suitable for the clinic. Therefore, PCR, a more specific and sensitive technique than the other methods, has become a preferred technique for diagnosing T. whipplei infection (13). In this report, we detected the presence of $P$. jirovecii and T. whipplei by NGS using BAL. NGS technology has been booming since 2014 for pathogenic testing (14). Nowadays, NGS has been widely applied to clinics.

Antibiotics including penicillin, streptomycin, tetracycline, ceftriaxone, meropenem, co-trimoxazole, doxycycline, and hydroxychloroquine have been used for the treatment of $T$. whipplei infection (13). In this case, oral sulfamethoxazoletrimethoprim treatment seems to be slow to work in the first few days. Adding intravenous meropenem for 1 week helped the patient to clinically improve.

Pneumocystis pneumonia is one of the common opportunistic infections in HIV-infected patients. This case highlights the occultation of acute T. whipplei pneumonia in an AIDS patient with PCP. Clinicians need to be alert to the occurrence of
T. whipplei infection and timely diagnosis and antibacterial treatments are essential.

\section{DATA AVAILABILITY STATEMENT}

The raw data supporting the conclusions of this article will be made available by the authors, without undue reservation.

\section{ETHICS STATEMENT}

The studies involving human participants were reviewed and approved by Ethics Committee of Xixi Hospital of Hangzhou. Written informed consent to participate in this study was provided by the participants' legal guardian/next of kin. Written informed consent was obtained from the individual(s), and minor(s)' legal guardian/next of kin, for the publication of any potentially identifiable images or data included in this article.

\section{AUTHOR CONTRIBUTIONS}

MW, JY, and BZ were involved in the clinical management of this patient. JY, JS, BZ, and ZZ collected the data of the patient. SL and 
JQ contributed to manuscript preparation. MW wrote the article. All authors read and approved the final manuscript.

\section{FUNDING}

This work was funded by Key projects of the Ministry of Science and Technology (No. 2018YFC1705701) and the Technology Plan of Medicine Health Science in Zhejiang Province (No. 2019KY533). The funding body

\section{REFERENCES}

1. Schmidt JJ, Lueck C, Ziesing S, Stoll M, Haller H, Gottlieb J, et al. Clinical course, treatment and outcome of Pneumocystis pneumonia in immunocompromised adults: a retrospective analysis over 17 years. Crit Care. (2018) 22:307. doi: 10.1186/s13054-018-2221-8

2. Xiao J, Gao G, Li Y, Zhang W, Tian Y, Huang Y, et al. Spectrums of opportunistic infections and malignancies in HIV-infected patients in tertiary care hospital, China. PLoS ONE. (2013) 8:e75915. doi: 10.1371/journal.pone.0075915

3. Raoult D. From whipple disease to Tropheryma whipplei infection. Clin Infect Dis. (2019) 68:1098-9. doi: 10.1093/cid/ciy668

4. Wang M, Dai X, Huang Y, Sun J, Dong X, Guo Y, et al. The presence of Pneumocystis jirovecii DNA in plasma is associated with a higher mortality rate in patients with AIDS-associated Pneumocystis pneumonia. Med Mycol. (2018). doi: 10.1093/mmy/myy110

5. Fenollar F, Laouira S, Lepidi H, Rolain JM, Raoult D. Value of Tropheryma whipplei quantitative polymerase chain reaction assay for the diagnosis of Whipple disease: usefulness of saliva and stool specimens for first-line screening. Clin Infect Dis. (2008) 47:659-67. doi: 10.1086/590559

6. Lozupone C, Cota-Gomez A, Palmer BE, Linderman DJ, Charlson ES, Sodergren E, et al. Widespread colonization of the lung by Tropheryma whipplei in HIV infection. Am J Respir Crit Care Med. (2013) 187:11107. doi: 10.1164/rccm.201211-2145OC

7. Stein A, Doutchi M, Fenollar F, Raoult D. Tropheryma whipplei pneumonia in a patient with HIV-2 infection. Am J Respir Crit Care Med. (2013) 188:10367. doi: 10.1164/rccm.201304-0692LE

8. Fenollar F, Ponge T, La Scola B, Lagier JC, Lefebvre M, Raoult D. First isolation of Tropheryma whipplei from bronchoalveolar fluid and clinical implications. J Infect. (2012) 65:275-8. doi: 10.1016/j.jinf.2011.1 1.026

9. Bassene H, Mediannikov O, Socolovschi C, Ratmanov P, Keita AK, Sokhna C, et al. Tropheryma whipplei as a Cause of Epidemic Fever, Senegal, 2010-2012. Emerg Infect Dis. (2016) 22:1229-334. doi: 10.3201/eid2207.150441

10. Masur H, Brooks JT, Benson CA, Holmes KK, Pau AK, Kaplan JE. Prevention and treatment of opportunistic infections in HIV-infected adults had no role in the design of the study and collection, analysis, interpretation of data, or preparation of the manuscript.

\section{ACKNOWLEDGMENTS}

The authors thank the staff in the HIV/AIDS ward of the Xixi Hospital of Hangzhou.

and adolescents: Updated Guidelines from the Centers for Disease Control and Prevention, National Institutes of Health, and HIV Medicine Association of the Infectious Diseases Society of America. Clin Infect Dis. (2014) 58:130811. doi: $10.1093 / \mathrm{cid} / \mathrm{ciu} 094$

11. Lagier JC, Papazian L, Fenollar F, Edouard S, Melenotte C, Laroumagne S, et al Tropheryma whipplei DNA in bronchoalveolar lavage samples: a case control study. Clin Microbiol Infect. (2016) 22:875-9. doi: 10.1016/j.cmi.2016.07.010

12. Esteves F, Lee CH, de Sousa B, Badura R, Seringa M, Fernandes C, et al. (13)-beta-D-glucan in association with lactate dehydrogenase as biomarkers of Pneumocystis pneumonia (PcP) in HIV-infected patients. Eur J Clin Microbiol Infect Dis. (2014) 33:1173-80. doi: 10.1007/s10096-014-2054-6

13. Dolmans RA, Boel CH, Lacle MM, Kusters JG. Clinical manifestations, treatment, and diagnosis of Tropheryma whipplei infections. Clin Microbiol Rev. (2017) 30:529-55. doi: 10.1128/CMR.00033-16

14. Wilson MR, Naccache SN, Samayoa E, Biagtan M, Bashir H, Yu G, et al. Actionable diagnosis of neuroleptospirosis by next-generation sequencing. $N$ Engl J Med. (2014) 370:2408-17. doi: 10.1056/NEJMoa1401268

Conflict of Interest: The authors declare that the research was conducted in the absence of any commercial or financial relationships that could be construed as a potential conflict of interest.

Publisher's Note: All claims expressed in this article are solely those of the authors and do not necessarily represent those of their affiliated organizations, or those of the publisher, the editors and the reviewers. Any product that may be evaluated in this article, or claim that may be made by its manufacturer, is not guaranteed or endorsed by the publisher.

Copyright (C) 2021 Yan, Zhang, Zhang, Shi, Liu, Qi and Wang. This is an open-access article distributed under the terms of the Creative Commons Attribution License (CC $B Y)$. The use, distribution or reproduction in other forums is permitted, provided the original author(s) and the copyright owner(s) are credited and that the original publication in this journal is cited, in accordance with accepted academic practice. No use, distribution or reproduction is permitted which does not comply with these terms. 